Results After adjustment for individual/neighbourhood socioeconomic variables, both outcomes were negatively associated with characteristics of the physical/service environments reflecting higher densities (eg, built surface area, street network connectivity, and densities of fruit/vegetables selling shops, fast-food restaurants, and healthcare resources). Multiple adjustment models were unable to disentangle the effects of these correlated densities. Analyses by pairs of participants similarly exposed to another environmental variable only identified a few associations, primarily with the density of fruit/vegetables selling shops.

Conclusion Overall, beyond influences of the socioeconomic environment, certain characteristics of the physical/service environments may be associated with weight status, but it may be difficult to disentangle the effects of various environmental dimensions because of the strong correlation between the variables (even if they imply different causal mechanisms and interventions).

\section{P1-212 HEALTH DISPARITIES AND SURVIVAL IN BRAZILIAN OLDER ADULTS}

doi:10.1136/jech.2011.142976e.5

M L Lebrão, * J L F Santos, Y Duarte. University of Sao Paulo, Sao Paulo, Brazil

Introduction Population ageing is not restricted to developed countries. In fact, the percentage of older adults is increasing more rapidly today in the developing world than in developed nations. These trends have important implications for understanding the mechanisms of population ageing. It will be impossible to understanding fully the global economic and political issues without an appreciation of the ageing of the world population. This study analysed health disparities associated with survival in older adult in a 6 year follow-up in Sao Paulo, Brazil.

Methods Data comes from a longitudinal survey-SABE Study (Health, Well-being and Ageing) that began in 2000 with a sample $(n=2.143$ ) of older adults ( $\geq 60$ years) living in São Paulo/Brazil. The $2^{\text {nd }}$ wave (2006) re-interviewed 1.115 elders. Multi variable analysis was performed adjusted logistic regression with robust estimation. Kaplan-Meier Survival Analysis was used to analyse death at 6 years.

Results Data show an annualised mortality rate of 55.2 per 1000 for males and 34.0 for females. The demographic variables associated with survival, besides age and gender, were greater education ( $p$ $<0.000)$, higher income $(p<0.00)$ and from an urban area in women $(p=0.015)$. The health related variables associated with survival were self-reported better health $(p<0.000$-women and $p=0.016$ men), no self-reported disease $(p<0.000)$, depression $(p=0.035$ for women) and no disability $(\mathrm{p}<0.000)$. In the Kaplan-Meier, men with excellent health are close to the women with regular health which is self-reported. Absence of disability makes the male curve higher than the female.

Conclusions Public policies should take into account the specific needs of the elderly population to facilitate access to healthcare services and reduce inequalities.

\section{P1-213 2009 PANDEMIC INFLUENZA A (H1N1) IN MALAYSIA}

doi:10.1136/jech.2011.142976e.6

${ }^{1} \mathrm{Y}$ L Lee, ${ }^{*} \mathrm{H}$ Ismail, ${ }^{3} \mathrm{~K}$ K Tan, ${ }^{1} \mathrm{P}$ Subramaniam, ${ }^{1} \mathrm{~T}$ Adnan, ${ }^{1} \mathrm{~J}$ Haniff. ${ }^{1} \mathrm{Clinical}$ Research Center, Ministry of Health Malaysia, Kuala Lumpur, Malaysia; ${ }^{2}$ Hospital Kuala Lumpur, Kuala Lumpur, Malaysia; ${ }^{3}$ Hospital Tuanku Ja'afar, Seremban, Malaysia

Background There is lack of children disease pattern data on children with 2009 pandemic influenza A(H1N1) in the tropics.
Methods We studied demographics, clinical features, and outcome of Malaysia hospitalised children aged 12 years and below with influenza A (H1N1) from $18^{\text {th }}$ June 2009 to $1^{\text {st }}$ March 2010. Real time web electronic case report form was used to collect data. We performed descriptive analysis and ORs with 95\% CI were calculated using logistic regression of binary outcome.

Results 1362 children with 2009 pandemic influenza A (H1N1) were hospitalised and $861(63.2 \%)$ were below 5 years. Children below 2 years accounted for over a third (39\%) of the patients admitted during the study and almost half (49\%) of the mortality. Of the 134 children admitted to the intensive care unit, 101 (75.4\%) required mechanical ventilation and 46 (34.3\%) died. Pneumonia and acute respiratory distress syndrome was present in 897 (85.5\%) and 41 (3.0\%) of the hospitalised children respectively including 18 of the $51(35.3 \%)$ non-survivors. The incidence of mortality for children below 12 years, below 5 years and below 2 years, between June through July 2009, was 0.1 per 100000 corresponding rates between August 2009 through February 2010 was $0.6,0.9$ and 1.3 per 100000 , respectively.

Conclusions The presence of one co-morbid was associated with higher rates of death and relatively a mild disease among hospitalised children in Malaysia.

\section{P1-214 ACCULTURATION, ETHNICITY AND ADOLESCENT MENTAL HEALTH IN THE UK: FINDINGS FROM THE DASH (DETERMINANTS OF ADOLESCENT SOCIAL WELL-BEING AND HEALTH) LONGITUDINAL STUDY}

doi:10.1136/jech.2011.142976e.7

${ }^{1} \mathrm{E}$ Lenguerrand, ${ }^{*}{ }^{2} \mathrm{~K}$ Bhui, ${ }^{1} \mathrm{H}$ Seeromanie, ${ }^{1} \mathrm{M}$ Maynard. ${ }^{1} \mathrm{MRC}$ SPSHU, University of Glasgow, Glasgow, UK; ${ }^{2}$ Centre for Psychiatry, Wolfson Institute of Preventive Medicine, Barts and the London School of Medicine \& Dentistry, London, UK

Background Acculturation is a stressful process of adaptation to societal changes and may influence cultural identity (eg, choice of friendships, religiosity). Ethnic minority boys in the UK, however, have a mental advantage over their White peers. Examining the relationship between cultural identity, ethnicity and psychological well-being may reveal mechanisms for protecting adolescent mental health.

Aim To examine the impact of cultural identity on psychological well-being in an ethnically diverse sample of adolescents.

Method Longitudinal data on psychological well-being (Goodman's Strengths and Difficulties Questionnaire), cultural identity (ethnicity of friendships, racism, migrant status) and potential correlates (eg, socio-economic circumstances) were collected on 4785 adolescents, $80 \%$ ethnic minorities, in 51 schools in London, UK. Ethnicity of friendships was used to classify pupils as integrated (many friends of own/other ethnicity), traditional (mostly friends of own ethnicity), assimilated (mostly friends of other ethnicity) and marginalised (few friends). Linear mixed models explored the impact of cultural identity on psychological well-being between 12 and $16 y$, and differential effects by ethnicity.

Results Psychological well-being improved through adolescence. Cultural identity was independently associated with psychological well-being among boys, with psychological well-being being most favourable in the integrated group and least so in the marginalised group. This effect did not vary by ethnicity or age and ethnic minority boys maintained a psychological well-being advantage over their White peers. Similar non-significant patterns were observed for girls.

Conclusion Acculturative processes impact on the psychological well-being of adolescent boys, regardless of ethnicity, and raises questions of how best to manage diversity in schools. 